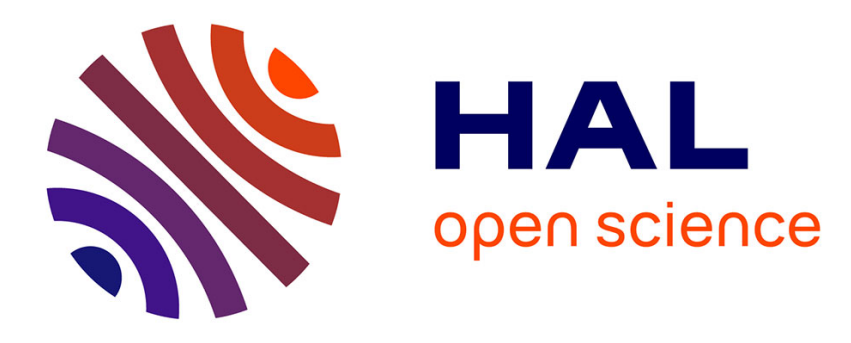

\title{
Pairing quadrupolaire et propriétés des états $(0+)$ des noyaux des terres rares
}

\author{
A. Issa
}

\section{To cite this version:}

A. Issa. Pairing quadrupolaire et propriétés des états $(0+)$ des noyaux des terres rares. Journal de Physique, 1981, 42 (2), pp.193-202. 10.1051/jphys:01981004202019300 . jpa-00209000

\section{HAL Id: jpa-00209000 https://hal.science/jpa-00209000}

Submitted on 1 Jan 1981

HAL is a multi-disciplinary open access archive for the deposit and dissemination of scientific research documents, whether they are published or not. The documents may come from teaching and research institutions in France or abroad, or from public or private research centers.
L'archive ouverte pluridisciplinaire HAL, est destinée au dépôt et à la diffusion de documents scientifiques de niveau recherche, publiés ou non, émanant des établissements d'enseignement et de recherche français ou étrangers, des laboratoires publics ou privés. 


\title{
Pairing quadrupolaire et propriétés des états $\left(0^{+}\right)$ des noyaux des terres rares
}

\author{
A. Issa $\left(^{+}\right)$ \\ Institut des Sciences Nucléaires, 38026 Grenoble Cedex, France
}

(Reçu le 10 juillet 1980, accepté le 16 octobre 1980)

\begin{abstract}
Résumé. - Le pairing quadrupolaire est introduit simultanément avec le pairing monopolaire et l'interaction à longue portée pour l'étude des états $\left(0^{+}\right)$excités dans la région des terres rares. Les calculs sont faits dans les approximations BCS et RPA. L'influence du choix des paramètres sur les résultats est discutée. Une attention particulière a été portée sur les états $\left(0^{+}\right)$possédant un rapport $X_{0}$ des probabilités de transition (E0) sur (E2) supérieur à 1 . Le pairing quadrupolaire est nécessaire pour expliquer les propriétés de ces états et il peut en outre expliquer l'apparition d'un troisième état $\left(0^{+}\right)$excité en dessous du gap.
\end{abstract}

\begin{abstract}
The quadrupole pairing is introduced simultaneously with monopole and quadrupole particle-hole interaction for the study of the excited $\left(0^{+}\right)$states in the rare-earth region. The calculations are made in the framework of BCS and RPA. The influence of the choice of parameters on the results is discussed. Particular attention has been devoted to $\left(0^{+}\right)$states having a ratio $X_{0}$ of the transition probabilities (E0) over (E2) larger than 1 . The quadrupole pairing is necessary to explain the properties of these states and can explain the apparition of a third $\left(0^{+}\right)$ excited state below the gap.
\end{abstract}

1. Introduction. - Ces dernières années, plusieurs auteurs se sont intéressés à l'étude des états excités $\left(0^{+}\right)$ des noyaux pair-pair déformés. Les transitions (E2) de ces états vers ceux de la bande fondamentale sont faibles, alors que les transitions (E0) de ces états vers le fondamental sont souvent fortes. Dans la région des actinides, les résultats expérimentaux [1] abondent. Dans la région des terres rares, la plupart des noyaux pair-pair ont été étudiés [2-19]. Dans cette région plusieurs états $\left(0^{+}\right)$excités sont parfois trouvés en dessous du gap d'énergie, et les rapports $X_{0}$ des probabilités de transitions réduites (E0) et (E2) de certains états sont supérieures à 1 .

Pour expliquer les propriétés de ces états $\left(0^{+}\right)$ plusieurs hypothèses ont été avancées. On a surtout essayé de modifier l'Hamiltonien et en particulier l'interaction résiduelle. Une interaction de pairing monopolaire et une interaction quadrupolaire à longue portée [20] ne peuvent pas faire apparaître plus d'un niveau en dessous du gap, de même que l'introduction d'une interaction spin-orbite à deux corps [21]. L'introduction d'une interaction spinquadrupole [22] ou d'un terme hexadécapolaire dans

$\left(^{+}\right)$Adresse permanente : Université Libanaise, Faculté des Sciences, Hadeth, Beyrouth, Liban. la force multipolaire [23] peut expliquer l'apparition d'un second $\left(0^{+}\right)$en dessous du gap. Tout récemment, des interprétations des résultats expérimentaux pour les isotopes de $\mathrm{Sm}$ [19] et de W [10] ont eu des succès dans le cadre de l'approximation des bosons en interaction [24] traitée à l'aide de la théorie des groupes. Une modification du traitement du pairing a été faite dans les actinides [25] et les terres rares [26] où l'équation de Bethe-Salpeter remplace une RPA généralisée, mais les résultats donnent des $B(\mathrm{E} 2)$ trop petits.

L'idée d'introduire un pairing non constant, dépendant de l'orbite a été reprise par plusieurs auteurs. L'idée première est de R. E. Griffin et al. [27]. On suppose qu'il y a deux catégories de niveaux individuels $|v\rangle$, oblate et prolate, suivant leur pente dans un schéma de Nilsson, que les éléments de matrice du pairing sont les mêmes pour les états de même type et que ces éléments de matrice sont réduits d'un facteur $\varepsilon<1$ pour des états de types différents. On peut alors, dans ce modèle simple, engendrer deux états superfluides. Plusieurs auteurs [28] ont repris cette idée en faisant des calculs plus réalistes. Plus récemment, on a introduit le pairing quadrupolaire et on a trouvé qu'il était important pour l'étude des états $\left(1^{+}\right)$et les moments d'inertie [29], le back- 
bending [30], les états à plusieurs phonons [31] et les états $\left(0^{+}\right)$des actinides [32]. L'interaction de pairing est définie par [33] :

$$
H_{\mathrm{p}}=-\sum_{\lambda^{\prime} \pi} G_{\lambda}^{\pi} P_{\lambda \pi}^{\pi+} P_{\lambda \pi}^{\pi}
$$

où l'on retient les deux premiers termes : $\lambda=0$, le terme habituel de pairing monopolaire et $\lambda=2$, le terme de pairing quadrupolaire; $\pi$ est l'espèce de particules (proton ou neutron). $G_{0}^{\pi}$ et $G_{2}^{\pi}$ sont les constantes de pairing $\left(G_{0}^{\pi}, G_{2}^{\pi}>0\right)$. Pour les états $\left(0^{+}\right)$ que nous considérons on prend :

$$
P_{20}^{\pi+}=-\sum_{v} q_{v}^{\pi(2)} a_{v}^{\pi+} a_{-v}^{\pi+}
$$

où $q_{v}^{\pi(2)}$ est un élément de matrice individuel dont nous discuterons le choix. Les éléments de matrice du pairing sont alors (nous omettons l'indice $\pi$ ) :

$$
\left\langle v\left|H_{\mathrm{p}}\right| v\right\rangle=-G_{0}-G_{2} q_{v}^{(2)} q_{v^{\prime}}^{(2)} .
$$

On retrouve l'idée de Griffin : si $q_{v}^{(2)} q_{v^{\prime}}^{(2)}>0$ les éléments de matrice (3) sont grands, si $q_{v}^{(2)} q_{v^{\prime}}^{(2)}<0$ les deux termes dans (3) se détruisent partiellement.

Dans cet article, nous introduisons le pairing quadrupolaire pour l'étude des états $\left(0^{+}\right)$des noyaux pair-pair dans les terres rares. D'abord, nous écrivons le formalisme et les équations théoriques. Ensuite, nous examinons l'influence sur les résultats du choix des différents paramètres et de la manière de les introduire dans les calculs. Enfin, nous étudions plus particulièrement les niveaux qui ont un rapport $X_{0}>1$.

2. Théorie. - 2.1 Hamiltonien. - L'Hamiltonien modèle est la somme de trois parties :

$$
\mathscr{H}=H_{\varepsilon}+H_{\mathrm{p}}+H_{\mathrm{MP}}
$$

$H_{\varepsilon}$ est un champ moyen qui décrit le mouvement individuel des nucléons dans le noyau. $H_{\mathrm{p}}$ et $H_{\mathrm{MP}}$ sont deux types d'interaction résiduelle qui tiennent compte respectivement des corrélations de paires et de l'interaction à longue portée. $H_{\varepsilon}$ sera représenté par le potentiel de Nilsson [34] qui s'écrit en seconde quantification :

$$
H_{\varepsilon}=\sum_{\pi, v} \varepsilon_{v}^{\pi}\left(a_{v}^{\pi+} a_{v}^{\pi}+a_{-v}^{\pi+} a_{-v}^{\pi}\right)
$$

où la sommation sur $v$ porte sur les états où $k_{v}>0$. $H_{\mathrm{p}}$ est défini par (2) et (3), $H_{\mathrm{MP}}$ par :

$$
H_{\mathrm{MP}}=-\overline{\frac{1}{2}} \sum_{\pi, \pi^{\prime}, \lambda, \mu} \chi_{\lambda \mu}^{\pi \pi^{\prime}} R_{\lambda \mu}^{\pi+} R_{\lambda \mu}^{\pi}
$$

où nous ne retiendrons que les termes $\lambda=2, \mu=0$ et où $R_{20}^{n+}$ est un opérateur défini par :

$$
R_{20}^{\pi+}=\sum_{v, v^{\prime}>0} Q_{v v^{\prime}}^{\pi(2)}\left(a_{v}^{\pi+} a_{v^{\prime}}^{\pi}+a_{-v}^{\pi+} a_{-v}^{\pi}\right) .
$$

$Q_{v v^{\prime}}^{\pi(2)}$ est l'élément de matrice quadrupolaire :

$$
Q_{v v^{\prime}}^{\pi(2)}=\left\langle v_{\pi}^{\prime}\left|r^{2} Y_{20}\right| v_{\pi}\right\rangle .
$$

Nous prendrons $\chi_{\lambda \mu}^{\pi \pi^{\prime}}=\chi$ pour tout $\pi, \pi^{\prime}, \lambda, \mu$ et par la suite nous omettrons, pour simplifier, les indices $\pi$ et $\pi^{\prime}$.

2.2 Equations BCS. - Pour chaque espèce de particules, nous cherchons par la méthode variationnelle les valeurs propres de l'Hamiltonien

$$
H^{\prime}=H+H_{\mathrm{p}} .
$$

Pour ce faire, nous introduisons la transformation canonique de Bogolyubov qui permet d'écrire $H^{\prime}$ sous la forme :

$$
H^{\prime}=U+H_{11}+H_{20}+H_{40}+H_{22}+H_{31}
$$

le terme $H_{31}$ n'intervient pas dans notre cas, nous le négligeons. Les termes $H_{40}$ et $H_{22}$ seront traités avec l'interaction résiduelle $H_{\mathrm{MP}}$. En exigeant que $H_{20}=0$ (alors $H$ se réduit aux deux premiers termes) et que le nombre $n$ de particules est conservé en moyenne, on obtient les trois équations suivantes :

$$
\begin{gathered}
\Delta_{0}\left\{\frac{2}{G_{0}}-\sum_{v} \frac{1}{E_{v}}\right\}=\Delta_{2} \sum_{v} \frac{q_{v}}{E_{v}}\left\{\frac{2}{G_{0}}-\sum_{v} \frac{1}{E_{v}}\right\}\left\{\frac{2}{G_{2}}-\sum_{v} \frac{q_{v}^{2}}{E_{v}}\right\}=\left(\sum_{v} \frac{q_{v}}{E_{v}}\right)^{2} \\
n=\sum_{v}\left(1-\frac{\varepsilon_{v}^{\prime}-\lambda}{E_{v}}\right)
\end{gathered}
$$

où

$E_{v}=\sqrt{\left(\varepsilon_{v}^{\prime}-\lambda\right)^{2}+\Delta_{v}^{2}}$ est l'énergie de quasi-particule,

$\Delta_{v}=\Delta_{0}+q_{v}^{(2)} \quad \Delta_{2}$ est un gap dépendant de l'état individuel $|v\rangle$,

$\Delta_{0}=G_{0} \sum_{v} U_{v} V_{v}, \quad \Delta_{2}=G_{2} \sum_{v} q_{v}^{(2)} U_{v} V_{v}, \quad \varepsilon_{v}^{\prime}=\varepsilon_{v}-V_{v}^{2} \sum_{\lambda=0,2} G_{\lambda} q_{v}^{(\lambda)^{2}}$. 
2.3 Equations RPA. - Nous introduisons les opérateurs d'excitation élémentaire qui créent ou détruisent deux quasi-particules couplées à $k=0$

$$
A_{-v, v^{\prime}}^{+}=\alpha_{v^{\prime}}^{+} \alpha_{-v}^{+} \quad A_{-v, v^{\prime}}=\alpha_{v} \alpha_{v^{\prime}} .
$$

Nous introduisons aussi les opérateurs de création et de destruction d'un phonon :

$$
\begin{aligned}
O_{i}^{+} & =\sum_{\substack{a, b>0 \\
\pi}} X_{a b}^{\pi}(i) A_{-a b}^{\pi+}-Y_{a b}^{\pi}(i) A_{-b a}^{\pi} \\
O_{i} & =\sum_{\substack{a, b>0 \\
\pi}} X_{a b}^{\pi}(i) A_{-a b}^{\pi+}-Y_{a b}^{\pi}(i) A_{-b a}^{\pi} .
\end{aligned}
$$

$\mathrm{Si}\left|\psi_{0}\right\rangle$ est l'état fondamental du noyau, en utilisant l'approximation RPA, nous exigeons que :

$$
\begin{aligned}
O_{i}\left|\psi_{0}\right\rangle & =0 \\
O_{i}^{+}\left|\psi_{0}\right\rangle & =\left|\psi_{i}\right\rangle= \\
& =\text { un état excité du noyau d'énergie } \omega_{i}
\end{aligned}
$$

ce qui peut s'écrire :

$$
\left[\mathfrak{H e}, O_{i}^{+}\right]=\omega_{i} O_{i}^{+} \text {. }
$$

L'orthogonalité des états impose :

$$
\left[O_{i}, O_{j}^{+}\right]=\delta_{i j}
$$

Ces deux conditions (13) et (14) déterminent les $X_{a b}(i)$ et $Y_{a b}(i)$ ainsi que les énergies $\omega_{i}$ des états $\left(0^{+}\right)$ du noyau, elles conduisent à l'équation de dispersion généralisée :

$$
\frac{1}{2 \chi}=\sum_{\pi} \mathscr{F}_{\pi}(\omega)
$$

avec :

$$
\begin{aligned}
\mathcal{F}_{\pi}(\omega)=F_{\pi}(\omega)+\sum_{\lambda}\left\{S_{1}^{\pi}(\lambda, \omega) S Q^{\pi}(\lambda, \omega)+\right. \\
\left.+S_{2}^{\pi}(\lambda, \omega) S K^{\pi}(\lambda, \omega)\right\} \\
F(\omega)=\sum_{a, b} \frac{\left[Q_{a b}^{(2)}\right]^{2} f_{a b}^{2} E_{a b}}{E_{a b}^{2}-\omega^{2}} \\
S_{1}(\lambda, \omega)=\sum_{a} \frac{Q_{a a}^{(2)} f_{a a} g_{a a} q_{a}(\lambda)}{E_{a a}^{2}-\omega^{2}} \\
S_{2}(\lambda, \omega)=\omega \sum_{a} \frac{Q_{a a}^{(2)} f_{a a}^{2} q_{a}(\lambda)}{E_{a a}^{2}-\omega^{2}}
\end{aligned}
$$

$S Q(\lambda, \omega)$ et $S K(\lambda, \omega)$ sont deux matrices carrées $4 \times 4$ dont les éléments sont des quantités analogues à $S_{1}$ et $S_{2}$. Pour ne pas alourdir le texte, nous n'en donnerons pas la forme explicite. $X_{a b}(i)$ et $Y_{a b}(i)$ sont données par :

$$
\begin{aligned}
& X_{a b}(i)=\frac{R(i)}{2\left(E_{a b}-\omega_{i}\right)}\left\{Q_{a b}^{(2)} f_{a b}+\delta_{a b} \sum_{\lambda=0,2} q_{a}^{(\lambda)} S K^{(i)}(\lambda)+\delta_{a b} g_{a a} \sum_{\lambda=0,2} q_{a}^{(\lambda)} S Q^{(i)}(\lambda)\right\} \\
& Y_{a b}(i)=\frac{R(i)}{2\left(E_{a b}+\omega_{i}\right)}\left\{Q_{a b}^{(2)} f_{a b}-\delta_{a b} \sum_{\lambda=0,2} q_{a}^{(\lambda)} S K^{(i)}(\lambda) \delta_{a b} g_{a a} \sum_{\lambda=0,2} q_{a}^{(\lambda)} S Q^{(i)}(\lambda)\right\}
\end{aligned}
$$

où $R(i)$ est une quantité qui dépend de $S Q, S K$ et de termes analogues à $S_{1}$ et $S_{2}$, dont nous ne donnerons pas l'expression détaillée et où :

$$
\begin{aligned}
E_{a b} & =E_{a}+E_{b} \\
g_{a a} & =U_{a}^{2}-V_{a}^{2} \\
f_{a b} & =U_{a} V_{b}+U_{b} V_{a} .
\end{aligned}
$$

2.4 OPÉRATEuRs. - La probabilité réduite de transitions $B\left(\mathrm{E} 2, I_{\mathrm{i}} \rightarrow I_{\mathrm{f}}\right)$ est définie par :

$$
\begin{aligned}
B\left(\mathrm{E} 2, I_{\mathrm{i}} \rightarrow I_{\mathrm{f}}\right) & = \\
& =\sum_{M, M_{\mathrm{f}}}\left|\left\langle I_{\mathrm{f}} O M_{\mathrm{f}}\left|E 2^{\mu}(\mathrm{S})\right| I_{\mathrm{i}} O M_{\mathrm{i}}\right\rangle\right|^{2}
\end{aligned}
$$

où $E 2^{\mu}(\mathrm{S})$ est l'opérateur de transition électromagnétique E2 exprimé dans le système de laboratoire (S) :

$$
E 2^{\mu}(\mathbf{S})=\sum_{\mathbf{p}} e R_{\mathbf{p}}^{2} Y_{2}^{\pi}\left(\theta_{\mathbf{p}^{\prime}} \varphi_{\mathrm{p}}\right)
$$

où la somme s'étend sur tous les protons de coor- données $R_{\mathrm{p}}, \theta_{\mathrm{p}}$ et $\varphi_{\mathrm{p}}$ dans le système du laboratoire, $e$ la charge du proton.

$\mathrm{Si}$ on introduit des charges effectives $e_{\text {eff }}^{\pi}$ pour tenir compte de la troncature de la base des états individuels, et si on écrit l'opérateur E2 dans le système lié au noyau, on aboutit à l'expression suivante :

$$
B\left(\mathrm{E} 2,0_{\mathrm{i}}^{+} \rightarrow 2_{\mathrm{f}}^{+}\right)=R^{2}(i)\left[\sum_{\pi} e_{\pi} \mathscr{F}_{\pi}\left(\omega_{i}\right)\right]^{2} .
$$

Dans nos résultats $B(\mathrm{E} 2)$ sera exprimé en single particle units (S.P.U.) défini par :

$$
B(\mathrm{E} 2)_{\text {S.P.U. }}=\frac{5}{4 \pi}\left(\frac{3}{5} R_{0}^{2}\right)^{2} e^{2}
$$

où $R_{0}=1,23 A^{1 / 3}$ est le rayon nucléaire.

La probabilité de transition $B(\mathrm{E} 0)$ est définie par :

$$
B(\mathrm{E} 0)=\Omega \rho^{2}
$$

où $\Omega$ est un facteur tabulé [35] qui dépend peu des 
propriétés du noyau, $\rho$ est le paramètre qui donne les renseignements concernant la structure nucléaire :

$$
\rho=\left\langle v_{\mathrm{f}}|E 0| v_{\mathrm{i}}\right\rangle
$$

E0 est l'opérateur transition monopolaire électrique donné par:

$$
E 0=\sum_{\pi} \frac{e_{\pi}}{e} \sum_{i} \frac{r_{i}^{\pi 2}}{R_{0}^{2}} .
$$

Les calculs conduisent à :

$$
\rho=\frac{R(i)}{R_{0}^{2}} \sum_{\pi} \frac{e_{\pi}}{e} L_{\pi}\left(\omega_{i}\right)
$$

où $\mathfrak{L}_{\pi}\left(\omega_{i}\right)$ se déduit de $\mathcal{F}_{\pi}\left(\omega_{i}\right)$ en remplaçant $Q_{v v^{\prime}}^{(2)}$ par l'élément de matrice $\left\langle v\left|r^{2}\right| v^{\prime}\right\rangle$.

Pour limiter le nombre de paramètres nous adoptons la même charge effective pour $B(\mathrm{E} 0)$ et $B(\mathrm{E} 2)$ :

$$
e_{\mathrm{p}}=e+e_{\mathrm{eff}} \quad e_{\mathrm{n}}=e_{\mathrm{eff}} .
$$

Le rapport $X_{0}$ défini par [36] s'écrit alors :

$$
X_{0}=\frac{\rho^{2}}{B(\mathrm{E} 2)} \text {. }
$$

3. Choix des paramètres. - Pour étudier l'influence des différents paramètres sur les résultats nous avons effectué des calculs pour trois noyaux tests couvrant la région des terres rares : ${ }^{158} \mathrm{Gd}$, ${ }^{174} \mathrm{Hf}$ et ${ }^{182} \mathrm{~W}$. Ces noyaux ont été sélectionnés de façon à avoir des répartitions différentes des orbites prolate et oblate au voisinage de la surface de Fermi. Ainsi, dans le champ neutron on a :

- ${ }^{158} \mathrm{Gd}$ : surtout des orbites prolate,

- ${ }^{174} \mathrm{Hf}$ : des orbites prolate d'un côté et oblate de l'autre,

$-{ }^{182} \mathrm{~W}$ : surtout des orbites oblate avec quelques prolate.

Dans le champ proton, on a des orbites prolate pour ${ }^{158} \mathrm{Gd}$ et quelques oblate pour ${ }^{174} \mathrm{Hf}$ et ${ }^{182} \mathrm{~W}$.

Les calculs de ce chapitre sont effectués pour une valeur moyenne de la déformation $\varepsilon=0,25$ et en incluant 30 orbites également réparties de part et d'autre de la surface de Fermi.

$3.1 q_{v}$ ET $G_{0}$. Chacune de ces deux quantités est définie de deux façons que nous noterons (B) et (R) :

Choix (B) : pour $q_{v}$ c'est la définition habituelle

$$
q_{v}=\sqrt{\frac{16 \pi}{5}}\left\langle v\left|r^{2} Y_{20}\right| v\right\rangle
$$

pour $G_{0}$, on impose à la valeur moyenne des gaps $\Delta_{v_{1}}$ et $\Delta_{v_{2}}$ soit $\bar{\Delta}_{v}=\frac{1}{2}\left(\Delta_{v_{1}}+\Delta_{v_{2}}\right)$ d'être égale à la valeur $\Delta_{\exp }$ du gap tirée de l'expérience, où $v_{1}$ et $v_{2}$ sont les orbites $\left({ }^{1}\right) \frac{Z}{2}$ et $\frac{Z}{2}+1$ pour les protons et $\frac{N}{2}$ et $\frac{N}{2}+1$ pour les neutrons.

Choix (R) : définitions adoptées dans la référence [32] :

$$
q_{v}=\sqrt{4 \pi} \frac{\left\langle v\left|r^{2} Y_{20}\right| v\right\rangle}{\left\langle v\left|r^{2}\right| v\right\rangle}
$$

pour $G_{0}$ on impose à la plus petite énergie de quasiparticule $E_{v_{0}}$ d'être égale à $\Delta_{\text {exp }}$. Dans la plupart des cas, $v_{0}$ est situé au voisinage direct de la surface de Fermi.

Pour les deux choix, les valeurs des gaps $\Delta_{\exp }$ sont tirées de la référence [37] où elles sont calculées à partir d'une formule empirique de masse.

Nous avons alors quatre définitions possibles pour le couple $\left(q_{v}, G_{0}\right):(\mathrm{B}, \mathrm{B}),(\mathrm{B}, \mathrm{R}),(\mathrm{R}, \mathrm{B})$ et $(\mathrm{R}, \mathrm{R})$. Pour faire des comparaisons il faut prendre $G_{2} \neq 0$. Certains auteurs fixent la valeur de $G_{2}$ avec une dépendance en $A$ [38] ou en $G_{0}$ [39]. D'après des calculs effectués avec une interaction $\delta$ de surface il est plus raisonnable de prendre $G_{2} \simeq G_{0}$ [29, 40], aussi dans les calculs de ce chapitre nous fixons le rapport $G_{2} / G_{0}=1$ (où on s'est ramené à des quantités exprimées avec la même unité).

Tableau I. - Valeurs expérimentales de $\omega, B(\mathrm{E} 2)$, $\rho^{2}$ et $X_{0}$ concernant les états $\left(0^{+}\right)$excités du noyau ${ }^{158} \mathrm{Gd}$, comparées aux résultats des calculs théoriques faits pour les quatre choix du couple $\left(q_{v}, G_{0}\right)$ et en fixant les paramètres suivants : $G_{2} / G_{0}=1, \varepsilon=0,25$, $e_{\mathrm{eff}} / e=0,2$; le taux de transition $B(\mathrm{E} 2)$ est exprimé en "Single Particle Unit» (S.P.U.) et $\omega$ en MeV.

[Experimental values of $\omega, B(\mathrm{E} 2), \rho^{2}$ and $X_{0}$ for the $0^{+}$ excited states of ${ }^{158} \mathrm{Gd}$ as compared to the results of the calculation with the four choices for the set $\left(q_{v}, G_{0}\right)$ but with a fixed value of $G_{2} / G_{0}=1, \varepsilon=0.25$, $e_{\text {eff }} / e=0.2$. The transition rate $B(\mathrm{E} 2)$ is expressed in single particle units (S.P.U.) and $\omega$ in $\mathrm{MeV}$.]

$\begin{array}{ccllll} & \text { Exp. [3] } & (\mathrm{R}, \mathrm{B}) & \mathbf{( B ,} \mathrm{B}) & \mathbf{( B ,}, \mathrm{R}) & \mathbf{( R , R )} \\ \omega\left(0_{1}^{+}\right) & - & - & - & - & - \\ B(\mathrm{E} 2) & 1,196 & 1,196 & 1,196 & 1,196 & 1,196 \\ \rho^{2} & - & 1,19 & 1,14 & 1,25 & 1,27 \\ X_{0} & - & 0,054 & 0,054 & 0,056 & 0,057 \\ \omega\left(0_{2}^{+}\right) & - & 0,32 & 0,33 & 0,32 & 0,32 \\ B(\mathrm{E} 2) & 1,452 & 1,729 & 1,639 & 1,781 & 1,802 \\ \rho^{2} & 0,025 \pm 0,004 & 0,044 & 0,071 & 0,24 & 0,083 \\ X_{0} & 0,97 & 0,003 & 0,012 & 0,004 \\ \omega\left(0_{3}^{+}\right) & \pm 0,12 & 0,32 & 0,34 & 0,37 & 0,35 \\ B(\mathrm{E} 2) & 1,743 & 1,777 & 1,699 & 1,854 & 1,851 \\ \rho^{2} & - & 0,074 & 0,16 & 0,15 & 0,078 \\ X_{0} & - & 0,005 & 0,010 & 0,010 & 0,005 \\ & - & 0,032 & 0,43 & 0,44 & 0,49 \\ & & & & & \end{array}$

( $\left.{ }^{1}\right)$ Une définition analogue a été utilisée par Hamamoto [29] où $v_{1}$ et $v_{2}$ sont les orbites des états fondamentaux des noyaux impairs $(A-1)$ et $(A+1)$ où $A=(Z, N)$ est le noyau pair-pair étudié. 
Les tableaux I, II et III montrent les résultats des calculs effectués avec les quatre définitions du couple $\left(q_{v}, G_{0}\right)$. Pour les trois noyaux étudiés, le cas $G_{0} \equiv(R)$ donne le plus grand écart avec les énergies expérimentales, $G_{0} \equiv(\mathrm{B})$ donne trois niveaux dans ${ }^{158} \mathrm{Gd}$ et deux niveaux dans ${ }^{174} \mathrm{Hf}$ en dessous du gap comme c'est le cas expérimentalement. On remarque en outre que le choix (R) ou (B) de $q_{v}$ n'affecte que très peu les résultats et que le choix $(\mathrm{B})$ de $G_{0}$ donne une meilleure stabilité des résultats. Le choix $(R, R)$ peut modifier considérablement les résultats dans les noyaux où existe un mélange d'orbites prolate et oblate au voisinage de la surface de Fermi.

D'autre part, le fait de fixer le rapport $G_{2} / G_{0}=1$ conduit à une renormalisation de la valeur de $G_{0}$ obtenue pour $G_{2}=0$. Avec le choix (R, B), alors que pour $G_{2}=0, G_{0}(\mathrm{p})$. varie entre $25,5 . A$ et $28,5 . A$

Tableau II. - Même légende que pour le tableau I, avec des valeurs des paramètres $G_{2} / G_{0}, \varepsilon$ et $e_{\text {eff }}$ identiques, pour le noyau ${ }^{174} \mathrm{Hf}$.

[Same caption as for table I, with the same values for the parameters $G_{2} / G_{0}, \varepsilon$ and $e_{\text {eff }}$, for ${ }^{174} \mathrm{Hf}$.]

$\begin{array}{ccllll} & \text { Exp. [6, 7] } & (\mathrm{R}, \mathrm{B}) & (\mathrm{B}, \mathrm{B}) & \mathbf{( B ;}, \mathrm{R}) & (\mathrm{R}, \mathrm{R}) \\ \omega\left(0_{1}^{+}\right) & - & - & - & - & - \\ B(\mathrm{E} 2) & 0,828 & 0,828 & 0,828 & 0,828 & 0,828 \\ \rho^{2} & 4 & 3,01 & 2,86 & 4,17 & 3,78 \\ X_{0} & 0,17, \leqslant 0,71 & 0,15 & 0,15 & 0,22 & 0,19 \\ \omega\left(0_{2}^{+}\right) & 1,241 & 1,418 & 1,391 & 1,557 & 1,534 \\ B(\mathrm{E} 2) & - & 0,57 & 0,75 & 0,15 & 0,13 \\ \rho^{2} & - & 0,033 & 0,043 & 0,011 & 0,010 \\ X_{0} & - & 0,41 & 0,40 & 0,51 & 0,55 \\ \omega\left(0_{3}^{+}\right) & - & 1,618 & 1,609 & 1,682 & 1,637 \\ B(\mathrm{E} 2) & - & 0,018 & 0,050 & 0,12 & 0,11 \\ \rho^{2} & - & 0,003 & 0,005 & 0,003 & 0,002 \\ X_{0} & - & 1,07 & 0,72 & 0,18 & 0,16\end{array}$

Tableau III. - Même légende que pour le tableau I, avec des valeurs des paramètres $G_{2} / G_{0}$, $\varepsilon$ et $e_{\text {eff }}$ identiques, pour le noyau ${ }^{182} \mathrm{~W}$.

[Same caption as for table I, with the same values for the parameters $G_{2} / G_{0}, \varepsilon$ and $e_{\mathrm{eff}}$, for ${ }^{182} \mathrm{~W}$.]

$\begin{array}{ccllll} & \text { Exp. [6, 7] } & (\mathrm{R}, \mathrm{B}) & (\mathrm{B}, \mathrm{B}) & (\mathrm{B}, \mathrm{R}) & (\mathrm{R}, \mathrm{R}) \\ \omega\left(0_{1}^{+}\right) & - & - & \cdot \overline{1} & - & - \\ B(\mathrm{E} 2) & 1,138 & \mathbf{1 , 1 3 8} & 1,138 & 1,138 & 1,138 \\ \rho^{2} & - & 2,44 & 2,75 & 2,26 & 2,12 \\ X_{0} & - & 0,14 & 0,16 & 0,13 & 0,12 \\ \omega\left(0_{2}^{+}\right) & 2,240 & 0,41 & 0,41 & 0,40 & 0,39 \\ B(\mathrm{E} 2) & - & 0,617 & 1,595 & 1,548 & 1,516 \\ \rho^{2} & - & 0,005 & 0,0005 & 0,004 & 0,011 \\ X_{0} & - & 0,25 & 0,34 & 0,35 & 0,56 \\ \omega\left(0_{3}^{+}\right) & 2,730 & 1,773 & 1,771 & 1,656 & 1,635 \\ \bar{B}(\mathrm{E} 2) & - & 0,056 & 0,072 & 0,023 & 0,009 \\ \rho^{2} & - & 0,001 & 0,001 & 0,003 & 0,0003 \\ X_{0} & - & 0,17 & 0,08 & 0,92 & 0,25\end{array}$

et $G_{0}(\mathrm{n})$ entre $23 . A$ et $24,5 . A$; pour $G_{2} / G_{0}=1$ on obtient une stabilité pour les trois noyaux étudiés :

$$
G_{0}(\mathrm{p}) \simeq 26,5 . A \text { et } G_{0}(\mathrm{n}) \simeq 20,5 . A .
$$

En conclusion de cette étude, nous ne garderons par la suite que le choix $(\mathrm{R}, \mathrm{B})$ pour le couple $\left(q_{v}\right.$, $G_{Q}$ ).

3.2 INFLUENCE D'UNE VARIATION DE $\boldsymbol{\Delta}$. - En l'absence d'orbites oblate au voisinage de la surface de Fermi $\left({ }^{158} \mathrm{Gd}\right)$ une variation de $10 \%$ de $\Delta_{\mathrm{p}}$ ou $\Delta_{\mathrm{n}}$ n'affecte pratiquement pas les résultats : $\omega, B(\mathrm{E} 2)$, $\rho$ et $X_{0}$. Dans ${ }^{174} \mathrm{Hf}$ et ${ }^{182} \mathrm{~W}$ où des orbites oblate apparaissent, les propriétés des états $\left(0^{+}\right)$ne sont pas très affectées mais on peut avoir à partir d'une certaine valeur de l'énergie des interversions de niveaux de même nature.

3.3 CUt-OFF. - Pour tenir compte de la troncature de la base des états de particules nous avons introduit une charge effective $e_{\text {eff }}$, celle-ci peut être considérée comme nulle dans le cas où tout le champ de particules est inclus. L'effet du cut-off est négligeable sur les énergies des états $\left(0^{+}\right)$et sur leur fonction d'onde. Par contre, pour avoir les mêmes valeurs pour $\rho$ et $B(\mathrm{E} 2)$ il faut augmenter la charge effective au fur et à mesure que la troncature est plus sévère. Ainsi pour 40 niveaux, il faut prendre $e_{\text {eff }} / e$ entre 0,01 et 0,1 et pour 30 niveaux il faut prendre $e_{\text {eff }} / e$ de l'ordre de 0,2 .

3.4 Déformation De la BaSE. - Nous avons effectué tous les calculs de manière cohérente dans la base cylindrique dépendant de la déformation $\left|\mathrm{NN}_{\mathrm{z}} \Lambda \Sigma(\varepsilon)\right\rangle$ définie par Boisson-Piepenbring [41]. La différence entre ce traitement et celui de Nilsson [34] réside ici dans le calcul des éléments de matrice de $r^{2}$ et de $r^{2} Y_{20}$, donc dans celui des éléments $q_{v}$. Les traitements de la référence [41] et de Nilsson conduisent dans certains cas à des natures d'états $\left(0^{+}\right)$et à des propriétés électromagnétiques de ces états différentes. D'une façon générale les $B(\mathrm{E} 0)$ et $B(\mathrm{E} 2)$ sont plus grandes dans le traitement de Boisson-Piepenbring que dans celui de Nilsson.

3.5 Paramètre $\chi$ De LA Force a LONGUe PORTÉE. Habituellement, et c'est ce que nous avons fait, la valeur de $\chi$ est choisie de manière à faire coïncider l'énergie du premier état $\left(0^{+}\right)$excité avec sa valeur expérimentale. Avec la valeur óbtenue de $\chi$ on calcule ensuite les autres $\left(0^{+}\right)$. Avec cette méthode, pour chaque valeur du rapport $G_{2} / G_{0}$ on a une valeur différente de $\chi$. Mais on peut aussi fixer la valeur de $\chi$, pour toute valeur de $G_{2} / G_{0}$, soit.en prenant celle obtenue avec la première méthode pour $G_{2}=0$ soit en adoptant une dépendance en $A[30,32]$. Le rapport $G_{2} / G_{0}$ étant fixé, nous avons étudié l'influence d'une variation de $\chi$ : les énergies et les fonc- 
tions d'onde des états $\left(0^{+}\right)$excités ne sont pratiquement pas touchées sauf pour le premier $\left(0^{+}\right)$excité où on peut parfois avoir des variations considérables avec $\chi$. Quant à $B(\mathrm{E} 0)$ et $B(\mathrm{E} 2)$ on peut retrouver les mêmes valeurs en changeant $e_{\text {eff }}$ : selon le sens de variation de $\chi$ il faut augmenter ou diminuer $e_{\text {eff }}$ pour retrouver les mêmes $B(\mathrm{E} 0)$ et $B(\mathrm{E} 2)$.

4. Etude des noyaux où on a observé des $X_{0}>1$. L'étude précédente sur l'influence des différents paramètres nous a indiqué le meilleur choix pour le couple $\left(q_{v}, G_{0}\right) \equiv(\mathrm{R}, \mathrm{B})$. Quant aux autres paramètres, nous avons vu que les différentes définitions et méthodes utilisées ne donnent pas des écarts supérieurs à $10 \%$ pour les quantités qui nous intéressent ici.

Dans ce chapitre, les discussions sont axées sur les seuls noyaux possédant des états $\left(0^{+}\right)$ayant un rapport $X_{0}>1$. Ils peuvent être classés en deux groupes : ${ }^{164} \mathrm{Er},{ }^{168} \mathrm{Yb}$ et ${ }^{172} \mathrm{Hf}$ d'une part et ${ }^{172} \mathrm{Yb}$ et ${ }^{176} \mathrm{Hf}$ de l'autre. Remarquons qu'à l'intérieur de chaque groupe, les noyaux se déduisent les uns des autres par le transfert d'une particule $\alpha$.

Le paramètre de déformation $\varepsilon$ peut varier pour chaque noyau selon les auteurs et les méthodes d'obtention $[15,20,37,42]$. Dans les calculs de ce chapitre, le critère du choix de $\varepsilon$ était de retrouver la situation expérimentale où dans certains noyaux plusieurs états $\left(0^{+}\right)$sont en dessous du gap. La figure 1 montre les courbes correspondantes où les énergies présentent un minimum, la situation expérimentale est reproduite (sauf pour ${ }^{176} \mathrm{Hf}$ ) autour de ce minimum qui se trouve dans un petit intervalle sur l'axe des $\varepsilon$. Les valeurs que nous adoptons sont :

$$
\begin{aligned}
& \varepsilon\left({ }^{164} \mathrm{Er}\right)=0,27, \quad \varepsilon\left({ }^{168} \mathrm{Yb}\right)=0,30, \\
& \varepsilon\left({ }^{172} \mathrm{Hf}\right)=0,33, \quad \varepsilon\left({ }^{172} \mathrm{Yb}\right)=0,275 \text {, } \\
& \varepsilon\left({ }^{176} \mathrm{Hf}\right)=0,32 \text {. }
\end{aligned}
$$

Avec ces valeurs de $\varepsilon$, la variation de l'énergie $\omega\left(0_{i}^{+}\right)$en fonction du rapport $G_{2} / G_{0}$ est montrée sur la figure 2. Alors que le pairing monopolaire seul $\left(G_{2}=0\right)$ ne peut donner qu'un seul niveau $\left(0^{+}\right)$ excité en dessous du gap, l'addition du pairing quadrupolaire $\left(G_{2} \neq 0\right)$ peut faire apparaître deux ou trois états $\left(0^{+}\right)$excités en dessous du gap. Remarquons qu'en introduisant d'autres forces résiduelles supplémentaires, les auteurs des références [22] et [23] ont pu obtenir un second $\left(0^{+}\right)$excité en dessous du gap mais jamais trois comme c'est le cas ici avec $G_{2} / G_{0}=1$.

Les tableaux IV à VIII montrent les résultats de nos calculs comparés à ceux de l'expérience; nous y avons reporté aussi les résultats disponibles d'autres calculs effectués sur ces noyaux. Les états où $X_{0}>1$

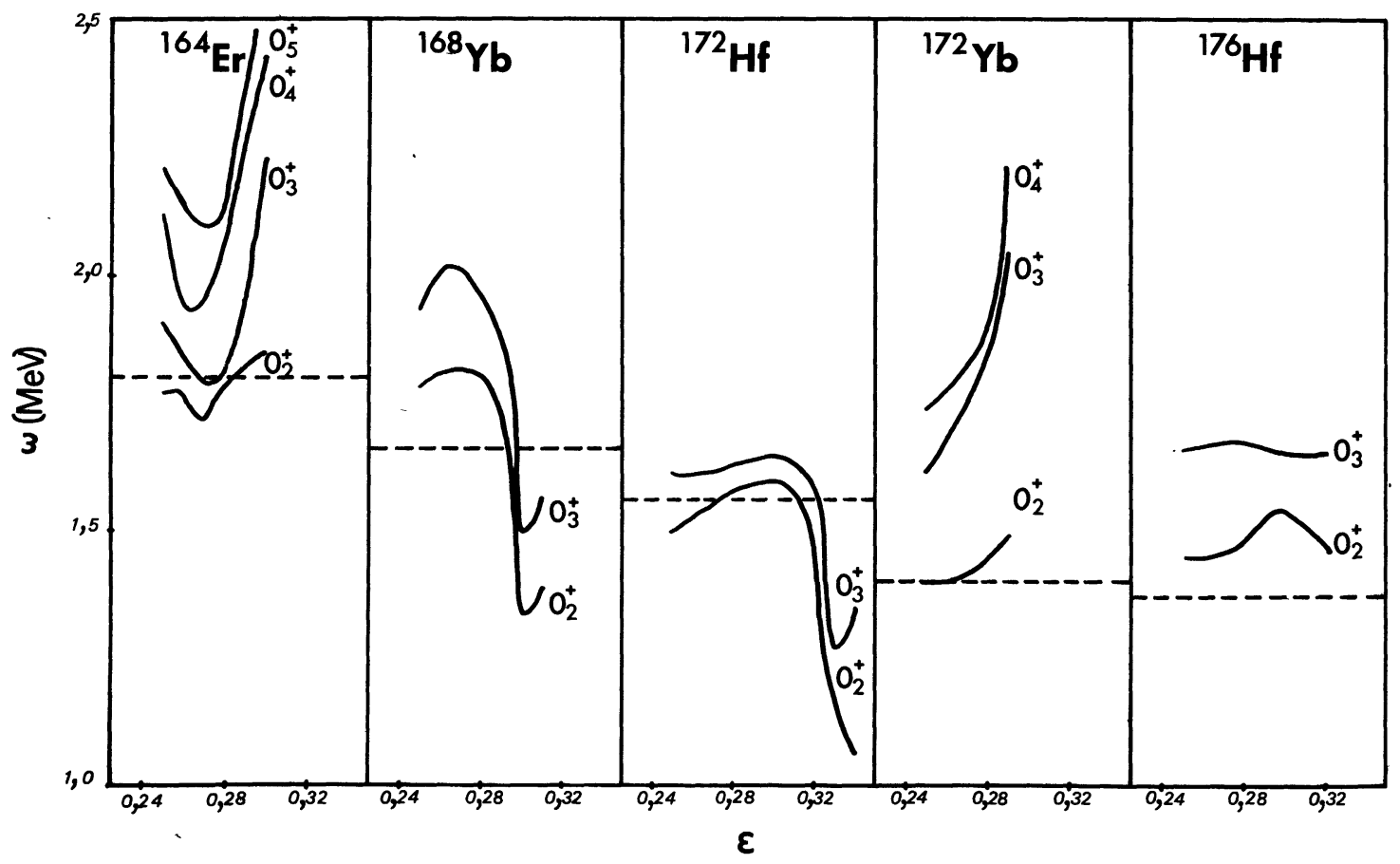

Fig. 1. - Courbes de variation des énergies $\omega$ des premiers états $\left(0^{+}\right)$excités en fonction du paramètre $\varepsilon$ de déformation pour les noyaux : ${ }^{164} \mathrm{Er},{ }^{168} \mathrm{Yb},{ }^{172} \mathrm{Hf},{ }^{172} \mathrm{Yb}$ et ${ }^{176} \mathrm{Hf}$. L'énergie du premier état $\left(0^{+}\right)$excité de chaque noyau n'y figure pas, elle coïncide avec la valeur expérimentale de l'énergie de cet état pour tout $\varepsilon$. Les calculs sont faits avec le choix $(\mathrm{R}, \mathrm{B})$ du couple $\left(q_{v}, G_{0}\right)$ et en fixant le rapport $G_{2} / G_{0}=1$. Les droites horizontales en pointillés représentent $2 \Delta_{\text {exp }}$, le double du gap expérimental de chaque noyau.

[Variations of the energies $\omega$ of the first $\left(0^{+}\right)$excited states as a function of the deformation parameter $\varepsilon$ for ${ }^{164} \mathrm{Er},{ }^{168} \mathrm{Yb},{ }^{172} \mathrm{Hf},{ }^{172} \mathrm{Yb}$ and ${ }^{176} \mathrm{Hf}$. The energy of the first $\left(0^{+}\right)$excited state is not given : it is identical to the experimental value for any $\varepsilon$. The calculations are done with the choice (R, B) for $\left(q_{v}, G_{0}\right)$ with a fixed ratio $G_{2} / G_{0}=1$. The horizontal dashed lines correspond to $2 \Delta_{\text {exp }}$, twice the experimental gap for each nucleus.] 


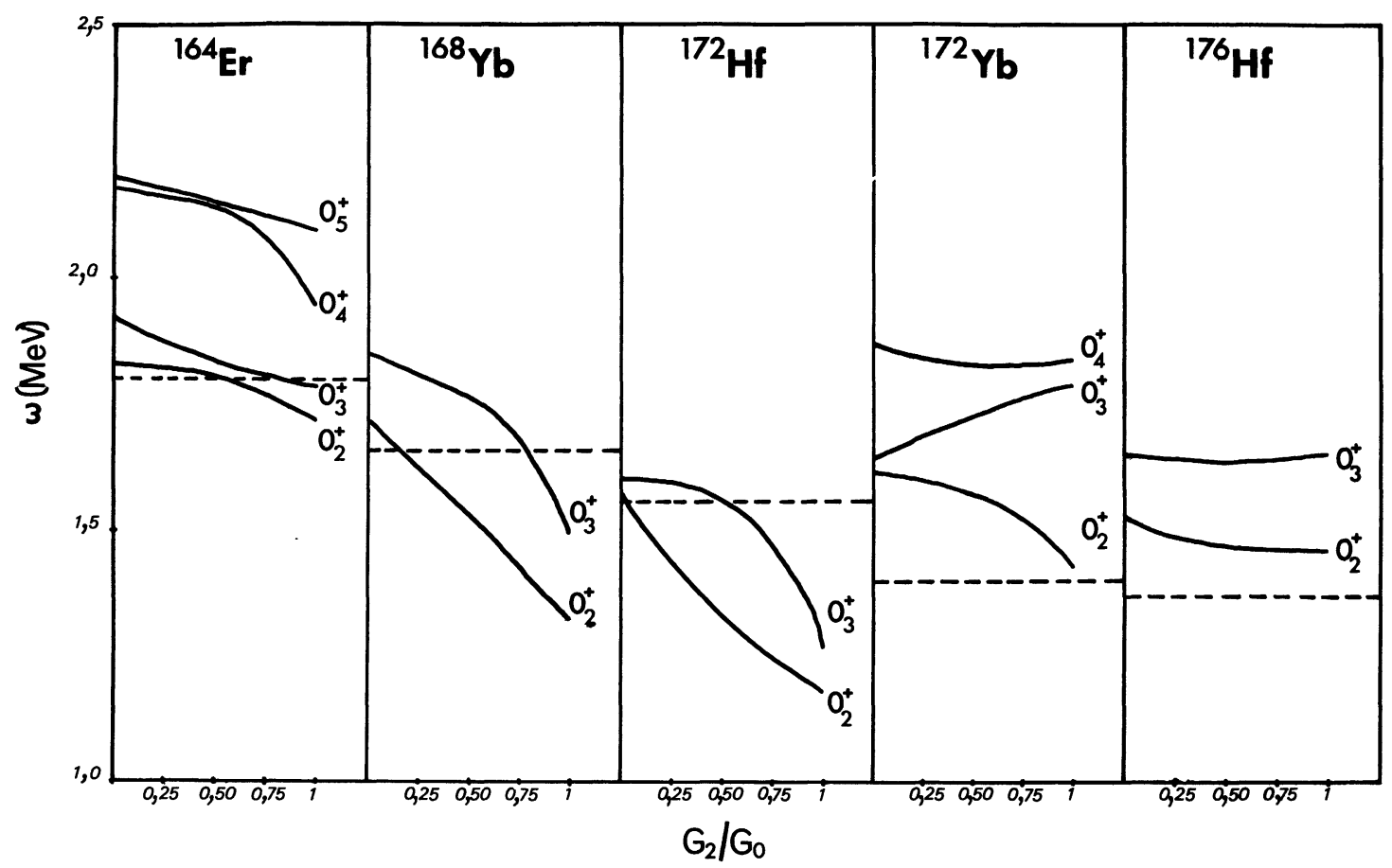

Fig. 2. - Courbes de variation des énergies $\omega$ des premiers états $\left(0^{+}\right)$excités en fonction du rapport $G_{2} / G_{0}$ variant entre 0 et 1 . Les calculs sont faits avec le choix $(\mathrm{R}, \mathrm{B})$ du couple $\left(q_{v}, G_{0}\right)$ et en fixant le paramètre de déformation $\varepsilon$ de chaque noyau (pour les valeurs de $\varepsilon$ voir le texte). L'énergie du premier état $\left(0^{+}\right)$excité de chaque noyau n'y figure pas, elle coïncide avec la valeur expérimentale de l'énergie de cet état pour toute valeur du rapport $G_{2} / G_{0}$.

[Variations of the energies $\omega$ of the first $\left(0^{+}\right)$excited states as a function of the ratio $G_{2} / G_{0}$ which is varied from 0 to 1 . The calculations are done with the choice $(\mathrm{R}, \mathrm{B})$ for $\left(q_{v}, G_{0}\right)$ with a fixed value of the deformation parameter $\varepsilon$ for each nucleus (see text for the values of $\varepsilon$ ). The energy of the first $\left(0^{+}\right)$excited state is not given : it is identical to the experimental value for all values of the ratio $G_{2} / G_{0}$.]

Tableau IV. - Valeurs expérimentales de $\omega, B(\mathrm{E} 2), \rho^{2}$ et $X_{0}$ concernant les états $\left(0^{+}\right)$excités du noyau ${ }^{164} \mathrm{Er}$, comparées aux résultats des calculs théoriques utilisant des interactions résiduelles différentes. Colonne $1:$ spinquadrupole [12], colonne 2 : terme hexadécapolaire [23], colonne $3: \delta$ de surface [26], colonnes 4 et $5:$ nos calculs faits respectivement avec $G_{2}=0$ et $G_{2} / G_{0}=1$ et pour $e_{\mathrm{eff}} / e=0,2$ et $\varepsilon=0,27$.

[Experimental values of $\omega, B(\mathrm{E} 2), \rho^{2}$ and $X_{0}$ for the $0^{+}$excited states of ${ }^{164} \mathrm{Er}$ as compared to the results of the calculation with various residual interactions. Col. $1:$ spin-quadrupole [12], col. 2 : hexadecapolar term [23], col. 3 : surface $\delta$ interaction [26], cols. 4 and 5 : our calculations with respectively $G_{2}=0$ and $G_{2} / G_{0}=1$, for $e_{\text {eff }} / e=0.2$ and $\varepsilon=0.27$.]

$\begin{array}{lcccccc} & 1 & 2 & 3 & 4 & 5 & \text { Exp. [6, 11, 12] } \\ \omega\left(0_{1}^{+}\right) & 1,25 & 1,246 & 1,27 & 1,246 & 1,246 & - \\ B(\mathrm{E} 2) & 0,98 & 1,50 & 0,40 & 2,21 & 1,00 & 1,246 \\ \rho^{2} & 0,02 & - & 0,04 & 0,10 & 0,05 & >10^{-4} ;>4 \times 10^{-4} \\ X_{0} & 0,11 & - & 0,70 & 0,31 & 0,34 & 0,15 \pm 0,03 \\ \omega\left(0_{2}^{+}\right) & 1,64 & 1,785 & 1,42 & 1,828 & 1,719 & 1,702 \\ B(\mathrm{E} 2) & 1,23 & - & 1,41 & 1,01 & 1,13 & - \\ \rho^{2} & 0,04 & - & 0,04 & 9 \times 10^{-4} & 0,01 & - \\ X_{0} & 0,22 & - & 0,70 & 0,69 & 0,49 & 0,39 \pm 0,06 \\ \omega\left(0_{3}^{+}\right) & 2,00 & - & - & 1,924 & 1,787 & 1,766 \\ B(\mathrm{E} 2) & 0,06 & - & - & 0,02 & 9 \times 10^{-5} & - \\ \rho^{2} & 0,0016 & - & - & 2 \times 10^{-4} & 9 \times 10^{-4} & - \\ X_{0} & 0,23 & - & - & 0,09 & 71,38 & 0,76 \pm 0,11 \\ \omega\left(0_{4}^{+}\right) & 2,11 & - & - & 2,177 & 1,945 & 2,172 \\ B(\mathrm{E} 2) & 0,50 & - & - & 4 \times 10^{-4} & 0,16 & - \\ \rho^{2} & 0,026 & - & - & 2 \times 10^{-4} & 2 \times 10^{-3} & - \\ X_{0} & 0,37 & - & - & 2,83 & 0,08 & 1,76 \pm 0,25 \\ \omega\left(0_{5}^{+}\right) & - & - & - & 2,199 & 2,095 & 2,185 \\ B(\mathrm{E} 2) & - & - & - & 0,05 & 9 \times 10^{-3} & - \\ \rho^{2} & - & - & - & 3 \times 10^{-4} & 3 \times 10^{-3} & - \\ X_{0} & - & - & - & 0,04 & 2,12 & 5,6 \pm 1,84 \\ & & & - & & & \end{array}$


Tableau V. - Même légende que pour le tableau IV, pour le noyau ${ }^{168} \mathrm{Yb}$ avec $\varepsilon=0,30$.

[Same caption as for table IV, for ${ }^{168} \mathrm{Yb}$ with $\varepsilon=0.30$.]

$\begin{array}{lcccccc} & 1 & 2 & 3 & 4 & 5 & \text { Exp. [6, 13] } \\ \omega\left(0_{1}^{+}\right) & - & - & \frac{-}{15} & - & - & - \\ B(\mathrm{E} 2) & 1,15 & 1,156 & 0,97 & 1,156 & 1,156 & 1,156 \\ \rho^{2} & 2,03 & 1,46 & 0,29 & 3,10 & 1,37 & >36 \times 10^{-4} ; 0,95 \\ X_{0} & 0,03 & - & 0,017 & 0,18 & 0,096 & 7 \times 10^{-5} \\ \omega\left(0_{2}^{+}\right) & 0,10 & - & 0,42 & 0,41 & 0,49 & 0,095-0,13 \\ B(\mathrm{E} 2) & 1,37 & 1,623 & 1,26 & 1,722 & 1,328 & 1,197 \\ \rho^{2} & 0,55 & - & 0,19 & 0,016 & 8 \times 10^{-3} & 6 \times 10^{-5} \\ X_{0} & 0,014 & - & 0,014 & 2 \times 10^{-3} & 7 \times 10^{-3} & <0,013 \\ \omega\left(0_{3}^{+}\right) & 0,16 & - & 0,55 & 0,73 & 6,13 & >0,035-0,081) \\ B(\mathrm{E} 2) & 1,67 & - & - & 1,853 & 1,495 & >1,1 ; 0,51 \\ \rho^{2} & <0,01 & - & - & 0,22 & 0,17 & <10^{-5} \\ X_{0} & >\times 10^{-4} & - & - & 5 \times 10^{-3} & 0,017 & <4 \times 10^{-5} \\ & >1,00 & - & - & 0,16 & 0,72 & >12 \times 10^{-6}\end{array}$

Tableau VI. - Même légende que pour le tableau IV, pour le noyau ${ }^{172} \mathrm{Hf}$ avec $\varepsilon=0,33$.

[Same caption as for table IV, for ${ }^{172} \mathrm{Hf}$, with $\varepsilon=0.33$.]

\begin{tabular}{|c|c|c|c|c|c|c|}
\hline & 1 & 2 & 3 & 4 & 5 & Exp. [17] \\
\hline & & - & - & - & - & - \\
\hline$\omega\left(0_{1}^{+}\right)$ & - & - & - & 0,871 & 0,871 & 0,871 \\
\hline$B(\mathrm{E} 2)$ & - & - & - & 5,21 & 1,45 & - \\
\hline$\rho^{2}$ & - & - & - & 0,34 & 0,14 & - \\
\hline$X_{0}$ & - & - & - & 0,46 & 0,65 & $0,121 \pm 0,018$ \\
\hline$\omega\left(0_{2}^{+}\right)$ & - & - & - & 1,578 & 1,179 & 1,296 \\
\hline$B(\mathrm{E} 2)$ & - & - & - & $5 \times 10^{-3}$ & $3 \times 10^{-3}$ & - \\
\hline$\rho^{2}$ & - & - & - & 0,024 & $3 \times 10^{-3}$ & - \\
\hline$X_{0}$ & - & - & - & 37,43 & 7,39 & $19 \pm 4$ \\
\hline$\omega\left(0_{3}^{+}\right)$ & - & - & - & 1,606 & 1,272 & 1,336 \\
\hline$B$ (E2) & - & - & - & $2 \times 10^{-3}$ & 0,13 & - \\
\hline$\rho^{2}$ & - & - & - & $9 \times 10^{-4}$ & 0,012 & - \\
\hline$X_{0}$ & - & - & - & 4,06 & 0,66 & $6,0 \pm 0,7$ \\
\hline
\end{tabular}

ne sont pas toujours bien décrits, toutefois le pairing quadrupolaire les décrit mieux que les autres calculs. Nous remarquons que ces états sont surtout des états de vibration de paires de proton à l'exception du quatrième niveau dans ${ }^{172} \mathrm{Yb}$ et du cinquième dans ${ }^{164} \mathrm{Er}$ où on a un état de vibration de paires de neutrons. De plus dans les noyaux considérés on retrouve toujours dans la fonction d'onde des états de vibration de paires de protons l'orbite $1 / 2+[411]_{p}$ avec une forte composante. Contrairement aux affirmations de J. Larysz et al. [16] l'orbite $1 / 2-[541]_{\mathrm{p}}$ ne semble pas jouer un rôle prédominant dans la description des états $\left(0^{+}\right)$à fort $X_{0}$ dans ces noyaux.

Tableau VII. - Même légende que pour le tableau IV, pour le noyau ${ }^{172} \mathrm{Yb}$ avec $\varepsilon=0,275$.

[Same caption as for table IV, for ${ }^{172} \mathrm{Yb}$, with $\varepsilon=0.275$.]

$\begin{array}{lcccccc} & 1 & 2 & 3 & 4 & 5 & \text { Exp. [16] } \\ \omega\left(0_{1}^{+}\right) & 1,04 & 1,043 & 1,13 & 1,043 & 1,043 & - \\ B(\mathrm{E} 2) & 2,80 & 0,50 & 0,36 & 1,64 & 1,48 & 1,043 \\ \rho^{2} & 0,04 & - & 0,35 & 0,072 & 0,078 & (2,3 \pm 0,7) .10^{-3} \\ X_{0} & 0,10 & - & 0,70 & 0,31 & 0,37 & 0,28 \pm 0,004 \\ \omega\left(0_{2}^{+}\right) & 1,24 & 1,427 & 1,33 & 1,616 & 1,426 & 1,405 \\ B(\mathrm{E} 2) & 1,02 & - & 0,028 & 0,16 & 0,49 & - \\ \rho^{2} & 0,02 & - & 9 \times 10^{-4} & 5 \times 10^{-3} & 0,024 & <0,026 \\ X_{0} & 0,14 & - & 0,21 & 0,21 & 0,35 & 2,81 \pm 0,31 \\ \omega\left(0_{3}^{+}\right) & 1,69 & - & - & 1,642 & 1,788 & 1,794 \\ B\left(\mathrm{E}_{2}\right) & 0,05 & - & - & 0,16 & 54 \times 10^{-5} & - \\ \rho^{2} & 9 \times 10^{-4} & - & - & 10^{-3} & 2 \times 10^{-6} & - \\ X_{0} & 0,14 & - & - & 0,05 & 0,027 & 0,34 \pm 0,04 \\ \omega\left(0_{4}^{+}\right) & 1,89 & - & - & 1,868 & 1,840 & 1,895 \\ B(\mathrm{E} 2) & 0,01 & - & - & 0,17 & 10^{-4} & - \\ \rho^{2} & 9 \times 10^{-4} & - & - & 0,014 & 3 \times 10^{-4} & - \\ X_{0} & 1,15 & - & - & 0,56 & 21,14 & 0,10 \pm 0,03\end{array}$


Tableau VIII. - Même légende que pour le tableau IV, pour le noyau ${ }^{176} \mathrm{Hf}$ avec $\varepsilon=0,32$.

[Same caption as for table IV, for ${ }^{176} \mathrm{Hf}$, with $\varepsilon=0.32$.]

\begin{tabular}{|c|c|c|c|c|c|c|}
\hline & 1 & 2 & 3 & 4 & 5 & Exp. $[13,15,18]$ \\
\hline & - & - & - & - & - & - \\
\hline$\omega\left(0_{1}^{+}\right)$ & 1,15 & 1,150 & 1,11 & 1,150 & 1,150 & 1,150 \\
\hline$B(\mathrm{E} 2)$ & 1,60 & 1,51 & 1,67 & 1,2 & 0,32 & - \\
\hline$\rho^{2}$ & 0,023 & - & 0,10 & 0,068 & 0,017 & - \\
\hline$X_{0}$ & 0,09 & - & 0,43 & 0,39 & 0,37 & $\begin{array}{l}0,16 \pm 0,03 \\
0,29 \pm 0,11 \\
0,21 \pm 0,08\end{array}$ \\
\hline$\omega\left(0_{2}^{+}\right)$ & 1,30 & 1,368 & 1,37 & 1,530 & 1,458 & 1,293 \\
\hline$B(\mathrm{E} 2)$ & 1,46 & - & 0,25 & 0,65 & 0,17 & - \\
\hline$\rho^{2}$ & 0,032 & - & 0,02 & 0,017 & 0,022 & - \\
\hline$X_{0}$ & 0,15 & - & 0,56 & 0,19 & 0,93 & $\begin{array}{l}8,3 \pm 1,2 \\
8,3 \pm 1,0\end{array}$ \\
\hline$\omega\left(0_{3}^{+}\right)$ & 1,62 & - & 1,51 & 1,648 & 1,650 & 1,749 \\
\hline$B(\mathrm{E} 2)$ & 0,25 & - & 0,016 & 0,11 & 0,18 & - \\
\hline$\rho^{2}$ & $64 \times 10^{-4}$ & - & $16 \times 10^{-4}$ & 0,032 & 0,032 & - \\
\hline$X_{0}$ & 0,19 & - & $2 \times 10^{-5}$ & 2,22 & 1,27 & - \\
\hline
\end{tabular}

5. Conclusion. - En conclusion de ce travail, on peut dire que l'introduction du pairing quadrupolaire est nécessaire pour expliquer certaines propriétés des états $\left(0^{+}\right)$dans les noyaux de la région des terres rares, en particulier les $X_{0}>1$. Elle permet en outre d'expliquer l'apparition dans certains cas d'un troisième état $\left(0^{+}\right)$excité en dessous du gap. Enfin, il faut noter l'importance du choix de la définition de certains paramètres comme l'intensité $G_{0}$ de la force de pairing monopolaire et des éléments de matrice fixant le pairing quadrupolaire.

Remerciements. - Je tiens à remercier Mr R. Piepenbring pour ses conseils utiles et les discussions que j'ai eues avec lui, ainsi que Mr J. P. Boisson pour sa collaboration dans les programmes de calculs numériques.

\section{Bibliographie}

[1] Maher, J. V., et al., Phys. Rev. C5 (1972) 1380.

MAher, J. V., et al., Phys. Rev. C6 (1972) 358. Voir aussi références citées dans ces publications.

[2] Shahabuddin, M. A. M., et al., B.A.P.S. 24 (1979) 667. Anderson, D. L., et al., Phys. Rev. C18 (1978) 383. Spanhoff, R., et al., Phys. Rev. C18 (1978) 493. FlynN, E. R. and Burke, D. G., Phys. Rev. C17 (1978) 501

[3] Greenwood, R. C., et al., Nucl. Phys. A304 (1978) 327.

[4] Fleming, D. G., et al., Phys. Rev. C8 (1973) 806.

[5] Cardoso, M. H., et al., Z. Phys. A272 (1975) 13.

[6] Graetzer, R., et al., Nucl. Phys. 76 (1966) 1.

[7] Charvet, A., et al., J. Physique 32 (1971) 359.

[8] Mortensen, M. H., et al., Phys. Lett. 70B (1977) 17.

[9] Demidov, A. M., et al., Nucl. Phys. A237 (1975) 125.

[10] BetTs, R. R., and Mortensen, M. H., Phys. Rev. Lett. 43(1979) 616.

[11] VRzaL, J., Bull. Acad. Sci. U.S.S.R. 31 (1967) 599.

[12] Abdelvagabova, S. K., Bull. Acad. Sci. U.S.S.R. 37 (1973) 1007.

[13] Aldushchenkov, A. V., and Voinova, N. A., Nucl. Data Tables 11 (1972) 299.

[14] Dzhelepov, B. S., et al., Bull. Acad. Sci. U.S.S.R. 37 (1973) 1.

[15] Oотноudt, M. A., and Hintz, N. M., Nucl. Phys. A213 (1973) 221.

[16] Larysz, J., et al., Nucl. Phys. A309 (1978) 128.

[17] Cardoso, M. H., et al., Nucl. Phys. A205 (1973) 121.

[18] Kноо, T. L., et al., Nucl. Phys. A202 (1973) 289.
[19] SAHA, S., et al., Phys. Lett. 85B (1979) 215.

[20] BÈs, D. R., Nucl. Phys. 49 (1963) 544.

Bès, D. R. and Broglia, R. A., Nucl. Phys. 80 (1966) 289.

Baranger, M. and Kumar, K., Nucl. Phys. 110 (1968) 529.

Silvestre-Brac, B. and PiePenbring, R., Phys. Lett. 44B (1973) 357

[21] Belyaev, S. T., Mat. Fys. Kgl. Medd. Dan. Vid. Selsk 31 no 11 (1959).

[22] ZheleZnova, K. M., et al., Bull. Acad. Sci. U.S.S.R. 31 (1967) 546.

Kuliev, A. A. and Pyatov, N. I., Bull. Acad. Sci. U.S.S.R. 32 (1968) 767.

[23] Silvestre-Brac, B. and Piepenbring, R., Z. Phys. A 272 (1975) 89.

[24] Arima, A. and Iachello, F., Phys. Rev. C 16 (1977) 2085.

Scholten, O., Iachello, F. and Arima, A., Ann. Phys. N.Y. 115 (1978) 321 et références citées dans cette publication.

[25] Immele, J. D. and Struble, G. L., Lett. Nuovo Cimento 7 (1973) 41.

[26] Zawischa, D., Speth, J. and Pal, D., Nucl. Phys. A 311 (1978) 445.

[27] Griffin, R. E., et al., Phys. Lett. 36B (1971) 281.

[28] Van Ru, W. I. and Kahana, S. H., Phys. Rev. Lett. 28 (1972) 50.

Chasman, R. R., Phys. Rev. Lett. 28 (1972) 1275.

Glas, D. and Mosel, V., Nucl. Phys. A 216 (1973) 563.

[29] Намамото, I., Nucl. Phys. A 232 (1974) 445. 
[30] Wakai, M. and Faessler, A., Nucl. Phys. A 295 (1978) 86.

[31] Kishimoto, T. and Tamura, T., Nucl. Phys. A 270 (1976) 317.

[32] Ragnarsson, I. and Broglia, R. A., Nucl. Phys. A 263 (1976) 315.

[33] Bès, D. R. and Broglia, R. A., Phys. Rev. C 3 (1971) 2349.

[34] Nilsson, S. G., Mat. Fys. Medd. Dan. Vid. Selsk 29 no 16 (1955).

[35] Church, E. L. and Wesneser, J., Phys. Rev. 103 (1956) 1035.

[36] Rasmussen, J. O., Nucl. Phys. 19 (1960) 85.

[37] Prior, O., Boehm, F. and Nilsson, S. G., Nucl. Phys. A 110 (1968) 257.
[38] Vdovin, A. I., et al., Bull. Acad. Sci. U.S.S.R. 40 (1976) 2183. Rangacharyulu, C., et al., Lett. Nuovo Cimento 41 (1975) 95. Udagawa, T., et al., Phys. Lett. 35B (1971) 129.

[39] Dambarusen, D., Bull. Acad. Sci. U.S.S.R. 41 (1977) 1281.

[40] Broglia, R. A., Bès, D. R. and Nilsson, S. G., Phys. Lett. 50B (1974) 213.

[41] Boisson, J. P. and Piepenbring, R., Nucl. Phys. A 168 (1971) 385.

[42] LöBNER, K. E. G. and Honig, V., Nucl. Data Tables A 7 (1970) 505. 